\title{
Short-range order in linear systems
}

\author{
T. T. M. Võ \\ Department of Chemical Engineering, Rice University, Houston, Texas 77005 \\ L.-J. Chen \\ Department of Chemical Engineering, Rice University, Houston, Texas 77005 \\ and Department of Chemical Engineering, National Taiwan University, No. 1, Sec. 4, Roosevelt Road, \\ Taipei, Taiwan 106 \\ M. Robert ${ }^{\text {a) }}$ \\ Rice Quantum Institute, and Center for Nanoscale Science and Technology, and Department of Chemical \\ Engineering, Rice University, Houston, Texas 77005
}

(Received 4 February 2003; accepted 17 June 2003)

For linear systems in the continuum in which the particles interact with nearest-neighbor forces, the pair distribution function at short distances can be expressed exactly in a simple form. Results are given for various hard-core interaction potentials, in which the attractive potential is either constant (square-well potential), linear, or V-shaped. The second form is related to the depletion interaction. (C) 2003 American Institute of Physics. [DOI: 10.1063/1.1599272]

\section{INTRODUCTION}

One-dimensional continuous systems of hard rods with (or without) nearest-neighbor attractive interactions have been investigated for a long time. ${ }^{1-7}$ The main importance of these systems lies in the fact that in contrast to two- or threedimensional continuous systems, exact analytic results can be obtained for most quantities of interest, in particular, distribution (correlation) functions. ${ }^{8-14}$ Moreover, onedimensional systems provide valuable insight into the complex static and dynamic properties of higher dimensional systems, and enable approximate methods to be tested and developed for the latter. ${ }^{15-17}$ Particularly illuminating illustrations of this role played by linear systems are the manifold pioneering investigations of Percus, ${ }^{18-20}$ the study of Fisher and Widom ${ }^{21}$ of the long-range behavior of the pair distribution function, and the recent analysis of Lekkerkerker and Widom $^{22}$ of the depletion interaction in hard sphere mixtures.

Lattice systems are well-known to be much more amenable to exact analytic analysis than their continuous analogs. However, an extended hard core is needed in lattice systems to propagate the effect of the repulsive part of the potential on the distribution functions, ${ }^{23}$ and this then makes the theoretical analysis no simpler than that for systems in the continuum. ${ }^{21}$

In this work, a system of one-dimensional hard rods in the continuum interacting with nearest-neighbor attractive potentials of finite range is considered. While exact expressions of the pair distribution function for these systems (and hence, since the Kirkwood superposition approximation is exact for these models, for all $n$-body distribution functions) was derived 50 years ago by Salsburg et al. ${ }^{24}$ it has apparently not been noted that the short-range behavior of the pair distribution function can be expressed analytically in simple

\footnotetext{
a) Author to whom correspondence should be addressed. Electronic mail: mrobert@rice.edu
}

forms (the case of pure hard rods in the absence of any attraction has been solved a long time ago ${ }^{1}$ ). These forms are derived and used in this work to derive the exact analytic expression of the pair distribution function at short distances for three different attractive potentials: the square-well, linear, and V-shaped potentials. The second potential is shown to be related to the depletion interaction in linear systems.

\section{DERIVATION OF RESULTS}

Consider a system of $N$ identical particles of length (diameter) $a$, located at the points $R_{1}, \ldots, R_{N}$ on a line of length $L$. Assuming that the potential energy $\Phi_{N}$ is the sum of pair potentials between nearest-neighboring particles, and choosing

$$
0<R_{1}<R_{2}<\cdots<R_{N}<L,
$$

we have

$$
\Phi_{N}=\sum \sum_{i<j} \Phi\left(R_{i j}\right)=\sum_{j=1}^{N-1} \Phi\left(R_{j+1}-R_{j}\right),
$$

where $\Phi(R)$ is the interaction potential between nearestneighbor particles $j$ and $j+1$ respectively located at $R_{j}$ and $R_{j+1}$.

If one assumes that the walls act as particles identical to those of the system, fixed at $R=0$ and $R=L$, the total potential energy becomes

$$
\Phi_{N}=\Phi\left(R_{1}\right)+\sum_{j=1}^{N-1} \Phi\left(R_{j+1}-R_{j}\right)+\Phi\left(L-R_{N}\right) .
$$

$\Phi(R)$ is further assumed to satisfy
(a) $\lim _{R \rightarrow 0} \Phi(R)=\infty$,
(b) $\lim _{R \rightarrow \infty} \Phi(R)=0$. 
The average number density, i.e., the probability per unit length that any molecule will be at point $r$, is defined as ${ }^{24}$

$$
\rho^{(1)}(r)=\sum_{k=1}^{N}\left\langle\delta\left(R_{k}-r\right)\right\rangle_{\mathrm{Av}}=\left\langle\sum_{k=1}^{N} \delta\left(R_{k}-r\right)\right\rangle_{\mathrm{Av}} .
$$

Similarly, the average density of ordered pairs is given by ${ }^{24}$

$$
\rho^{(2)}\left(r, r^{\prime}\right)=\left\langle\sum_{k=1}^{N} \sum_{j=1 \neq k}^{N} \delta\left(R_{k}-r\right) \delta\left(R_{j}-r^{\prime}\right)\right\rangle_{\mathrm{Av}} ;
$$

here $\rho^{(2)}$ is the probability per (unit length) ${ }^{2}$ that one molecule will be at $r$ and another will be at $r^{\prime}$. For hard-core models with a hard-core diameter (length) $a$ and an attraction of range $\lambda$, the interaction potential takes the form,

$$
\Phi(R)=\left\{\begin{array}{l}
\infty \quad R<a \\
\Phi(R) \quad a \leqslant R \leqslant \lambda . \\
0 \quad R>\lambda
\end{array}\right.
$$

If the length of the system $L$ and the number of particles $N$ are allowed to become infinite in such a manner that $r-r^{\prime}$ $=R$ remains fixed (thermodynamic limit), the following expressions are obtained ${ }^{24}$

$$
\begin{aligned}
& \rho^{(1)}=1 / l a, \quad \text { with } l=L /[(N+1) a], \\
& \begin{aligned}
\rho^{(2)}(R)= & \sum_{n=1}^{N-1} \frac{A(R-n a)}{l a} \frac{1}{2 \pi i} \int_{c-\infty i}^{c+\infty i} d s \\
& \times\left[\frac{\Omega(s)}{\Omega(c)}\right]^{n} \exp [R(s-c)],
\end{aligned} \\
& \rho^{(2)}(R)=\rho^{(2)}(-R),
\end{aligned}
$$

where $A$ is the step function

$$
A(x)= \begin{cases}0 & x<0 \\ 1 & x \geqslant 0,\end{cases}
$$

$\Omega(s)$ is the Laplace transform

$$
\Omega(s)=\int_{0}^{\infty} d R \exp [-s R-\beta \Phi(R)]=\mathcal{L}\left[e^{-\beta \Phi(R)}\right]
$$

and $c$ is determined by solving the stationary condition for the saddle point ${ }^{24}$

$$
\left(\frac{\partial f}{\partial s}\right)_{s=c}=0
$$

where

$$
f(s)=\frac{L s}{N+1}+\ln \Omega(s) .
$$

For example, for a system of rigid spheres (rods) with no attraction, one readily finds $c=1 /[a(l-1)]$.

Consider next the short-range behavior of the pair distribution function $g(R)$, i.e., $n=1$ and $n=2$ in Eq. (6b), where $0 \leqslant R \leqslant 3 a$. For $n=1$, the first term of the sum in Eq. (6b) can be rewritten as

$$
\begin{aligned}
T_{1}(R) & =\frac{A(R-a)}{l a} \frac{1}{2 \pi i} \int_{c-i \infty}^{c+i \infty} d s e^{R s} \Omega(s) \frac{e^{-R c}}{\Omega(c)} \\
& =\frac{A(R-a)}{l a} \frac{e^{-R c}}{\Omega(c)} \mathcal{L}^{-1}\left\{\mathcal{L}\left[e^{-\beta \Phi(R)}\right]\right\} \\
& =\frac{A(R-a)}{l a} \frac{e^{-R c}}{\Omega(c)} e^{-\beta \Phi(R)} \\
& =\frac{A(R-a)}{l a} \frac{e^{-R c}}{\Omega(c)} I_{1}(R),
\end{aligned}
$$

where

$$
I_{1}(R)=e^{-\beta \Phi(R)} .
$$

Let

$$
I_{n}(R)=\frac{1}{2 \pi i} \int_{c-i \infty}^{c+i \infty} d s e^{R s}[\Omega(s)]^{n}
$$

so that

$$
I_{2}(R)=\frac{1}{2 \pi i} \int_{c-i \infty}^{c+i \infty} d s e^{R s}[\Omega(s)]^{2} .
$$

This integral is evaluated by using the relation ${ }^{6}$

$$
\int_{0}^{\infty} e^{-s t} f_{(m)}(t) d t=\left[\int_{0}^{\infty} e^{-s t} f(t) d t\right]^{m}
$$

where

$$
\begin{aligned}
f_{(N+1)}(u)= & \int_{0}^{u} d x_{N} \int_{0}^{x_{N}} d x_{N-1} \cdots \int_{0}^{x_{2}} f\left(u-x_{N}\right) \\
& \times f_{N, N-1} \cdots f_{21} f\left(x_{1}\right) d x_{1},
\end{aligned}
$$

with

$$
f_{N, N-1}=f\left(x_{N}-x_{N-1}\right)
$$

and

$$
f(u)=e^{-\beta \Phi(u)} .
$$

The integral in Eq. (12) then reads

$$
\begin{aligned}
I_{2}(R) & =\frac{1}{2 \pi i} \int_{c-i \infty}^{c+i \infty} d s e^{R s}\left[\int_{0}^{\infty} d R e^{-s R} e^{-\beta \Phi(R)}\right]^{2} \\
& =\frac{1}{2 \pi i} \int_{c-i \infty}^{c+i \infty} d s e^{R s}\left[\int_{0}^{\infty} d R e^{-s R} f_{(2)}(R)\right] \\
& =\int_{O}^{R} d R^{\prime} \exp \left\{-\beta\left[\Phi\left(R-R^{\prime}\right)+\Phi\left(R^{\prime}\right)\right]\right\},
\end{aligned}
$$


and the second term in Eq. (6b) thus becomes

$$
\begin{aligned}
T_{2}(R)= & \frac{A(R-2 a)}{l a} \frac{e^{-R c}}{[\Omega(c)]^{2}} \int_{0}^{R} d R^{\prime} \\
& \times \exp \left\{-\beta\left[\Phi\left(R-R^{\prime}\right)+\Phi\left(R^{\prime}\right)\right]\right\} . \\
= & \frac{A(R-2 a)}{l a} \frac{e^{-R c}}{[\Omega(c)]^{2}} I_{2}(R) .
\end{aligned}
$$

The average densities (3) and (4) in terms of $T_{1}(R)$ and $T_{2}(R)$ thus have the simple forms

$$
\rho^{(2)}(R)=T_{1}(R) \text { for } R \leqslant 2 a,
$$

and

$$
\rho^{(2)}(R)=T_{1}(R)+T_{2}(R) \text { for } R \leqslant 3 a,
$$

with $T_{1}$ and $T_{2}$ given by Eqs. (10a) and (17), respectively.

The pair distribution function $g^{(2)}$ is related to $\rho^{(2)}$ by

$g^{(2)}(R)=\rho^{(2)}(R)(l a)^{2}$.

We next apply the above results to derive the short-range behavior of the pair distribution function of the hard sphere (rod) model for three different attractive potentials: squarewell, linear, and V-shaped.

\section{A. Square-well potential}

The square-well potential is defined by

$$
\Phi(R)= \begin{cases}+\infty & R<a \\ -\epsilon & a \leqslant R \leqslant \lambda . \\ 0 & R>\lambda\end{cases}
$$

For $n=1$, the first term in Eq. (6b) becomes

$$
T_{1}(R)=\left\{\begin{array}{l}
0 \quad R<a \\
\frac{1}{l a} \frac{e^{-R c+\beta \epsilon}}{\Omega(c)} \quad a \leqslant R \leqslant \lambda . \\
\frac{1}{l a} \frac{e^{-R c}}{\Omega(c)} \quad \lambda<R
\end{array} .\right.
$$

For $n=2$, Eq. (16) can be rewritten as

$$
\begin{aligned}
I_{2}(R)= & \int_{0}^{a} d R^{\prime} \exp \left\{-\beta\left[\Phi\left(R-R^{\prime}\right)+\Phi\left(R^{\prime}\right)\right]\right\} \\
& +\int_{a}^{\lambda} d R^{\prime} \exp \left\{-\beta\left[\Phi\left(R-R^{\prime}\right)+\Phi\left(R^{\prime}\right)\right]\right\} \\
& +\int_{\lambda}^{R} d R^{\prime} \exp \left\{-\beta\left[\Phi\left(R-R^{\prime}\right)+\Phi\left(R^{\prime}\right)\right]\right\} .
\end{aligned}
$$

Two different interaction ranges are considered: $\lambda=2 a$ and $\lambda=3 a / 2$.

(i) $\lambda=2 a$ : Because of the nature of the square-well potential, the first and last terms in Eq. (20) vanish, resulting in

$$
I_{2}(R)=\int_{a}^{2 a} d R^{\prime} \exp \left[\beta \epsilon-\beta \Phi\left(R-R^{\prime}\right)\right]=y e^{2 \beta \epsilon},
$$

where $y+2 a=R$ with $0 \leqslant y \leqslant a$, and

$$
I_{2}(R)=(R-2 a) e^{2 \beta \epsilon} \text { for } 2 a \leqslant R \leqslant 3 a \text {. }
$$

$$
\begin{aligned}
I_{2}(R)= & \int_{a}^{3 a / 2} d R^{\prime} \exp \left\{-\beta\left[(-\epsilon)+\Phi\left(R-R^{\prime}\right)\right]\right\} \\
& +\int_{3 a / 2}^{5 a / 2} d R^{\prime} \exp \left[-\beta \Phi\left(R-R^{\prime}\right)\right] \\
& +\int_{5 a / 2}^{R} d R^{\prime} \exp \left[-\beta \Phi\left(R-R^{\prime}\right)\right]
\end{aligned}
$$

For the same reason as in (i) above, the last two terms vanish.

Let $x=R-R^{\prime}$ and $R=5 a / 2+y$, with $0 \leqslant y \leqslant a / 2$. One then finds

$$
\begin{aligned}
I_{2}(R)= & \int_{3 a / 2+y}^{a+y}-d x \exp \{-\beta[(-\epsilon)+\Phi(x)]\} \\
& -\int_{a+y}^{y} d x \exp [-\beta \Phi(x)] \\
= & e^{2 \beta \epsilon}(3 a-R)+2 e^{\beta \epsilon}\left(R-\frac{5}{2} a\right) .
\end{aligned}
$$

For $2 a \leqslant R \leqslant 5 a / 2$, letting $x=R-R^{\prime}$ and $R=2 a+y$, with $0 \leqslant y \leqslant a / 2$, we get

$$
\begin{aligned}
I_{2}(R)= & \int_{a}^{3 a / 2} d R^{\prime} \exp \left\{-\beta\left[(-\epsilon)+\Phi\left(R-R^{\prime}\right)\right]\right\} \\
& +\int_{3 a / 2}^{R} d R^{\prime} \exp \left[-\beta \Phi\left(R-R^{\prime}\right)\right] \\
= & \int_{a}^{a+y} d x \exp (2 \beta \epsilon)=(R-2 a) e^{2 \beta \epsilon}
\end{aligned}
$$

There remains to determine $\Omega(s)$. We have

$$
\begin{aligned}
\Omega(s)= & \int_{0}^{\infty} d R \exp [-s R-\beta \Phi(R)] \\
= & \int_{0}^{a} d R \exp [-s R-\beta \Phi(R)] \\
& +\int_{a}^{\lambda} d R \exp [-s R-\beta \Phi(R)] \\
& +\int_{\lambda}^{\infty} d R \exp [-s R-\beta \Phi(R)] \\
= & \frac{\exp (-\lambda s)}{s}[1-\exp (\beta \epsilon)]+\frac{1}{s} \exp (\beta \epsilon-s a) .
\end{aligned}
$$

$\Omega(c)$ is determined by solving Eqs. (9a), (9b), and (23). 


\section{B. Linear potential}

The linear potential has the form

$$
\Phi(R)=\left\{\begin{array}{l}
\left(\frac{\epsilon}{\lambda-a}\right) x-\frac{\lambda \epsilon}{\lambda-a}=\eta x-\xi \quad a \leqslant x \leqslant \lambda \\
+\infty \quad x<a \\
0 \quad x>\lambda
\end{array},\right.
$$

where

$$
\eta=\frac{\epsilon}{\lambda-a} \quad \text { and } \xi=\frac{\lambda \epsilon}{\lambda-a} .
$$

The expression of $\Omega(s)$ then takes the form,

$$
\begin{aligned}
\Omega(s)= & \int_{a}^{\lambda} d R \exp [-s R-\beta(\eta R-\xi)] \\
& +\int_{\lambda}^{\infty} d R \exp (-s R) \\
= & \frac{\exp (\beta \xi)}{(s+\beta \eta)}\{\exp [-(s+\beta \eta) a] \\
& -\exp [-(s+\beta \eta) \lambda]\}+\frac{1}{s} \exp (-\lambda s) .
\end{aligned}
$$

The expression $I_{1}(R)$ is determined as above. We obtain

$$
I_{1}(R)=\exp [-\beta(\eta R-\xi)\rfloor .
$$

The first term in Eq. (6b) then becomes

$$
T_{1}(R)=\left\{\begin{array}{lll}
0 & R<a \\
\frac{1}{l a} \frac{\exp (-R c)}{\Omega(c)} \exp [-\beta(\eta R-\xi)] \quad & a \leqslant R \leqslant \lambda \\
\frac{1}{l a} \frac{\exp (-R c)}{\Omega(c)} & \lambda<R
\end{array} .\right.
$$

For $n=2$, we again distinguish two cases:

(i) $\lambda=2 a$ : For $2 a \leqslant R \leqslant 3 a$,

$$
I_{2}(R)=\int_{a}^{2 a} d R^{\prime} \exp \left\{-\beta\left[\left(\eta R^{\prime}-\xi\right)+\Phi\left(R-R^{\prime}\right)\right]\right\} .
$$

Let $x=R-R^{\prime}$, and $y+2 a=R$, with $0 \leqslant y \leqslant a$. One finds

$$
\begin{aligned}
I_{2}(R) & =\int_{y}^{a+y} d x \exp \left\{-\beta\left[\left(\eta R^{\prime}-\xi\right)+\Phi(x)\right]\right\} \\
& =(R-2 a) \exp [-\beta(\eta R-2 \xi)] .
\end{aligned}
$$

(ii) $\lambda=3 a / 2$ : For $2 a \leqslant R \leqslant 5 a / 2$, we have

$$
I_{2}(R)=\int_{a}^{3 a / 2} d R^{\prime} \exp \left\{-\beta\left[\eta R^{\prime}-\xi+\Phi\left(R-R^{\prime}\right)\right]\right\} .
$$

Let $x=R-R^{\prime}$; then

$$
I_{2}(R)=\int_{R-a}^{R-3 a / 2}(-d x) \exp \left\{-\beta\left[\eta R^{\prime}-\xi+\Phi(x)\right]\right\} .
$$

Let next $R=2 a+y$, with $0 \leqslant y \leqslant a / 2$, so that

$$
\begin{aligned}
I_{2}(R) & =\int_{a / 2+y}^{a+y} d x \exp \left\{-\beta\left[\eta R^{\prime}-\xi+\Phi(x)\right]\right\}, \\
& =(R-2 a) \exp \lfloor-\beta(\eta R-2 \xi)\rfloor .
\end{aligned}
$$

For $5 a / 2 \leqslant R \leqslant 3 a, I_{2}(R)$ assumes the form,

$$
I_{2}(R)=\int_{a}^{3 a / 2} d R^{\prime} \exp \left\{-\beta\left[\eta R^{\prime}-\xi+\Phi\left(R-R^{\prime}\right)\right]\right\}
$$

$$
+\int_{3 a / 2}^{2 a} d R^{\prime} \exp \left[-\beta \Phi\left(R-R^{\prime}\right)\right] .
$$

Let $x=R-R^{\prime}$ and $R=5 a / 2+y$, with $0 \leqslant y \leqslant a / 2$. We get

$$
\begin{aligned}
I_{2}(R)= & \int_{3 a / 2+y}^{a+y}(-d x) \exp \left\{-\beta\left[\eta R^{\prime}-\xi+\Phi(x)\right]\right\} \\
& +\int_{a+y}^{a / 2+y}(-d x) \exp [-\beta \Phi(x)] \\
= & (3 a-R) \exp \{-\beta[\eta R-2 \xi]\} \\
& +\frac{\exp [\beta(-\eta R+\xi)]}{\beta \eta}\{\exp [\beta \eta(R-a)] \\
& \left.-\exp \left(\frac{3}{2} \beta \eta a\right)\right\}-\frac{\exp (\beta \xi)}{\beta \eta} \\
& \times\left\{\exp \left[-\beta \eta\left(R-\frac{3}{2} a\right]-\exp (-\beta \eta a)\right\} .\right.
\end{aligned}
$$

\section{V-shaped potential}

The V-shaped potential is defined as

$$
\Phi(x)=\left\{\begin{array}{l}
-\frac{2 \epsilon}{(\lambda-a)} x+\frac{2 \epsilon a}{(\lambda-a)} \quad a \leqslant x \leqslant \frac{(a+\lambda)}{2} \\
\frac{2 \epsilon}{(\lambda-a)} x-\frac{2 \epsilon \lambda}{(\lambda-a)} \quad \frac{1}{2}(a+\lambda) \leqslant x \leqslant \lambda
\end{array} .\right.
$$

Substituting Eq. (30) into Eq. (8) gives

$$
\begin{aligned}
\Omega(s)= & \int_{a}^{(a+\lambda) / 2} d R \exp \left[-s R-\beta\left(-\frac{2 \epsilon R}{\lambda-a}+\frac{2 \epsilon a}{\lambda-a}\right)\right] \\
& +\int_{(a+\lambda) / 2}^{\lambda} d R \exp \left[-s R-\beta\left(\frac{2 \epsilon R}{\lambda-a}-\frac{2 \epsilon \lambda}{\lambda-a}\right)\right] \\
& +\int_{\lambda}^{\infty} d R \exp (-s R) \\
= & \left\{\exp (-s a)-\exp \left[-\frac{(a+\lambda) s}{2}+\beta \epsilon\right]\right\} \\
& \times\left(s-\frac{2 \beta \epsilon}{\lambda-a}\right)^{-1}-\{\exp (-\lambda s) \\
& \left.-\exp \left[-\frac{s(a+\lambda)}{2}+\beta \epsilon\right]\right\}\left(s+\frac{2 \beta \epsilon}{\lambda-a}\right)^{-1} \\
& +\frac{\exp (-\lambda s)}{s} .
\end{aligned}
$$

For $n=1$, we obtain 


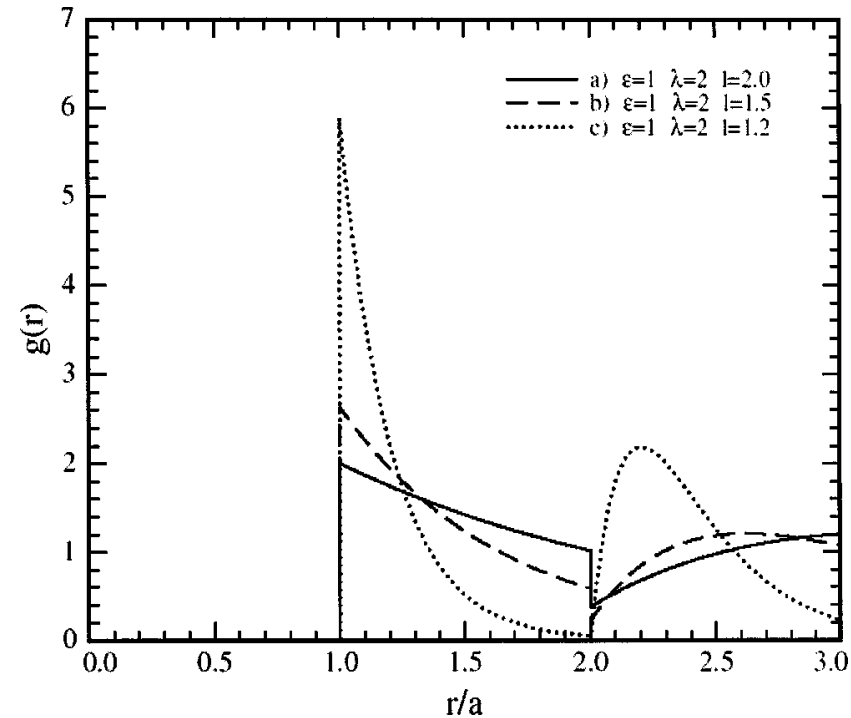

FIG. 1. Pair distribution function $g(r)=g^{(2)}(r)$ for the square-well potential for various values of $l$ and identical values of $\epsilon$ and $\lambda$. The units of $\epsilon$ are $k_{B} T$, and those of $\lambda$ and $l$ are $a$. The same units are used for the other figures.

$I_{1}(R)=\left\{\begin{array}{l}0 \quad R<a \\ \exp \left[-\beta\left(-\frac{2 \epsilon R}{\lambda-a}+\frac{2 \epsilon a}{\lambda-a}\right)\right] \quad a \leqslant R \leqslant \frac{1}{2}(a+\lambda) \\ \exp \left[-\beta\left(\frac{2 \epsilon R}{\lambda-a}-\frac{2 \epsilon \lambda}{\lambda-a}\right)\right] \quad \frac{1}{2}(a+\lambda) \leqslant R \leqslant \lambda \\ 1 \quad R>2 a\end{array}\right.$.

For $n=2, I_{2}(R)$ becomes

$$
\begin{aligned}
I_{2}(R)= & \int_{a}^{3 a / 2} d R^{\prime} \exp \left\{-\beta\left[\Phi\left(R^{\prime}\right)+\Phi\left(R-R^{\prime}\right)\right]\right\} \\
& +\int_{3 a / 2}^{2 a} d R^{\prime} \exp \left\{-\beta\left[\Phi\left(R^{\prime}\right)+\Phi\left(R-R^{\prime}\right)\right]\right\}
\end{aligned}
$$

Let

$$
\alpha=\frac{2 \epsilon}{\lambda-a}=\frac{2 \epsilon}{a},
$$

with $\lambda=2 a$. We have

$$
\begin{aligned}
I_{2}(R)= & \int_{a}^{3 a / 2} d R^{\prime} \exp \left\{-\beta\left[-\alpha R^{\prime}+2 \epsilon+\Phi\left(R-R^{\prime}\right)\right]\right\} \\
& +\int_{3 a / 2}^{2 a} d R^{\prime} \exp \left\{-\beta\left[\alpha R^{\prime}-4 \epsilon+\Phi\left(R-R^{\prime}\right)\right]\right\}
\end{aligned}
$$

For $2 a \leqslant R \leqslant 5 a / 2$, let $x=R-R^{\prime}$ and $y+2 a=R$, with $0 \leqslant y$ $\leqslant a / 2$; then

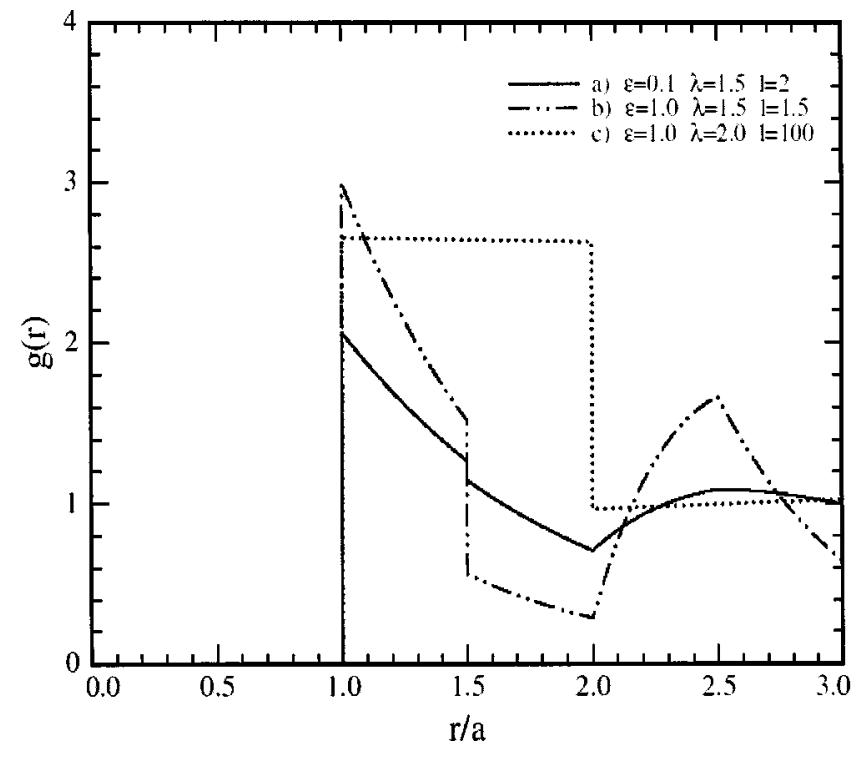

FIG. 2. Pair distribution function $g(r)$ for the square-well potential for various values of $\epsilon, \lambda$, and $l$.

$$
\begin{aligned}
I_{2}(R)= & \int_{R-a}^{R-3 a / 2}(-d x) \exp \left\{-\beta\left[-\alpha R^{\prime}+2 \epsilon+\Phi(x)\right]\right\} \\
& +\int_{R-3 a / 2}^{R-2 a}(-d x) \exp \left\{-\beta\left[\alpha R^{\prime}-4 \epsilon+\Phi(x)\right]\right\} \\
= & (R-2 a) \exp \left[\beta\left(\frac{2 \epsilon R}{a}-4 \epsilon\right)\right] .
\end{aligned}
$$

For $5 a / 2 \leqslant R \leqslant 3 a$, let $x=R-R^{\prime}$ and $y+5 a / 2=R$, with $0 \leqslant y \leqslant a / 2$; we have

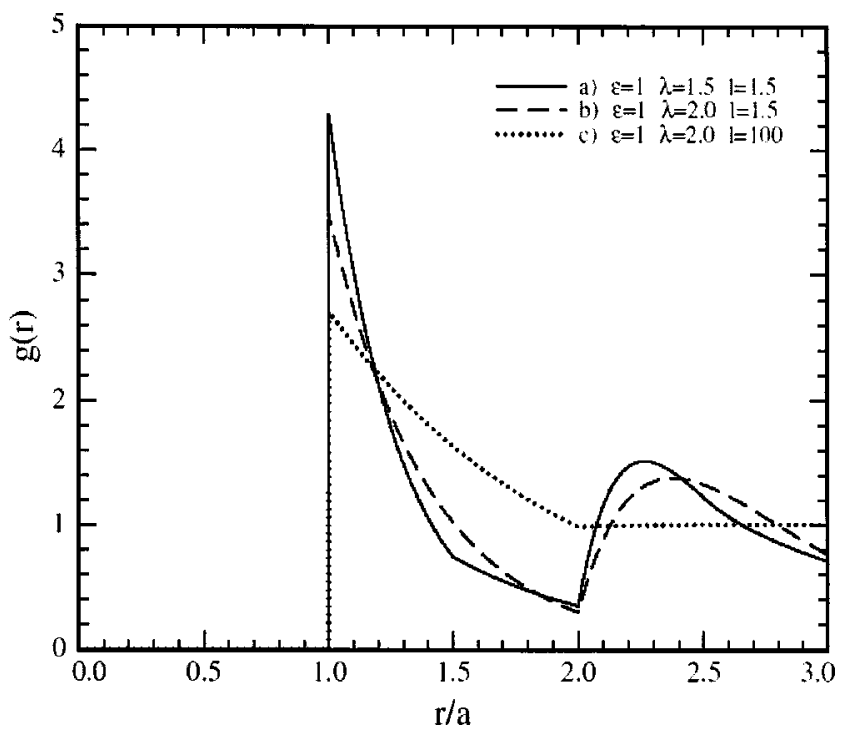

FIG. 3. Pair distribution function $g(r)$ for the linear potential for various values of $\epsilon, \lambda$, and $l$. 


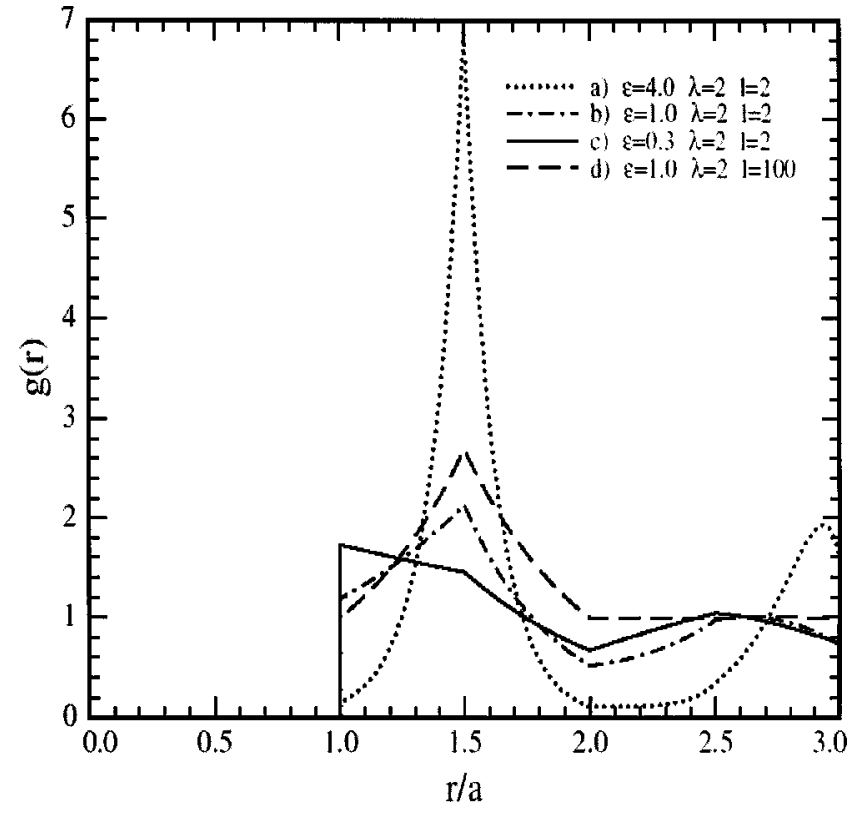

FIG. 4. Pair distribution function $g(r)$ for the V-shaped potential for various values of $\epsilon, \lambda$, and $l$.

$$
\begin{aligned}
I_{2}(R)= & \int_{y+a}^{y+3 a / 2} d x \exp \left\{-\beta\left[-\alpha R^{\prime}+2 \epsilon+\Phi(x)\right]\right\} \\
& +\int_{y+a / 2}^{y+a} d x \exp \left\{-\beta\left[\alpha R^{\prime}-4 \epsilon+\Phi(x)\right]\right\} \\
= & (3 a-R) \exp \left[\beta\left(\frac{2 \epsilon R}{a}-4 \epsilon\right)\right] \\
& +\left(R-\frac{5}{2} a\right) \exp \left[-\beta\left(\frac{2 \epsilon R}{a}-2 \epsilon\right)\right] \\
& +\frac{a}{4 \epsilon \beta}\left\{\exp \left[\beta\left(\frac{2 \epsilon R}{a}-4 \epsilon\right)\right]\right. \\
& \left.-\exp \left[\beta\left(-\frac{2 \epsilon R}{a}+6 \epsilon\right)\right]\right\} .
\end{aligned}
$$

\section{APPLICATIONS AND DISCUSSION}

The exact results derived above are used here to plot the short-range behavior of the pair distribution function for different values of the parameters of the intermolecular potentials and of the density. Figures 1 and 2 pertain to the squarewell potential, Fig. 3 pertains to the linear potential, and Fig. 4 to the V-shaped potential.

Noteworthy of these graphs is their apparently remarkable variety and occasionally surprising behavior. Several of these features are readily accounted for: in particular, the discontinuities of the first derivative of the pair distribution functions, clearly seen for example in Figs. 1, 2(a)-2(b), $3(a)-3(b)$, and 4(a)-4(c), are direct manifestations of those of the intermolecular potentials themselves, while the discontinuities of the pair distribution function itself, seen in Figs. 1(a)-1(b) and 2(a)-2(b), are direct manifestations of those of the potential itself (here the square-well potential); and for very dilute systems, i.e., for large values of the parameter $l$, the density expansion of the pair distribution function yields a short-range behavior which by necessity directly mimics the potential, as seen in Figs. 2(c), 3(c), and 4(d).

The pair distribution function generally attains its maximum value at contact, as seen for example in Figs. 1 and 2. However, when the well of the attractive part of the potential is deep and its minimum does not occur at contact, the maximum of the pair distribution function may occur away from contact. This is in accord with intuition, since the Boltzmann factor is largest at the well minimum. Such an instance is seen for the V-shaped potential in Figs. 4(a) and 4(b); it is not seen in Fig. 4(c), which corresponds to a shallower attractive potential minimum.

In its simplest approximation, i.e., that of Asakura and Oosawa, ${ }^{25}$ the depletion force is evaluated for a $d$-dimensional system of colloidal particles and ideal polymers, in which the polymer is modeled as a sphere of radius equal to its radius of gyration, and the colloid-polymer interaction is assumed to be hard spherelike. The depletion force is found to be proportional to the $(d-1)$-dimensional volume of the projection, on the plane perpendicular to the line joining the centers of two neighboring spherical particles, of the intersection of the excluded volumes of these particles. Therefore, for one-dimensional systems, the intersection reduces to a point, independently of the separation of the two colloidal particles provided that their centers are close enough to each other that no polymer can fit between them. Consequently, the depletion force is constant, and the depletion potential which is equal, up to the sign, to the integral of the depletion force, is a linear function of the separation between neighboring particles, precisely of the linear type studied here and in another recent study. ${ }^{26}$ In a forthcoming paper, the present exact results for the pair distribution functions will be compared to those of our ongoing experiments on one-dimensional colloid-polymer systems, and a qualitative comparison will be attempted with those of our experiments on two-dimensional colloid-polymer systems.

\section{ACKNOWLEDGMENTS}

This paper is dedicated to the memory of Zevi W. Salsburg, Professor of Chemistry at Rice University from 1956 until his untimely death at age 41 in 1970, on the occasion of the exact 50th anniversary of the submission to the Journal of Chemical Physics of his seminal work with John G. Kirkwood and Robert W. Zwanzig on one-dimensional distribution functions (Ref. 24). We are grateful to the National Science Foundation (Grant No. CTS-97000147) and the Welch Foundation (Houston, TX) for financial support, and to the National Science Council of Taiwan for making possible the visit of one of us (L.-J. C.) to Rice University, where the present work was performed. We thank Professor R. Curl for information on his former colleague Professor Z. Salsburg. 
${ }^{1}$ K. F. Herzfeld and M. Goeppert-Mayer, J. Chem. Phys. 2, 38 (1934).

${ }^{2}$ L. Tonks, Phys. Rev. 50, 955 (1936).

${ }^{3}$ T. Nagamiya, Proc. Phys. Math. Soc. Jpn. 22, 705 (1940); 22, 1034 (1940).

${ }^{4}$ J. Frenkel, Kinetic Theory of Liquids (Oxford University Press, New York, 1940), p. 126.

${ }^{5}$ G. Rushbrooke and H. Ursell, Proc. Cambridge Philos. Soc. 44, 263 (1948).

${ }^{6}$ F. Gürsey, Proc. Cambridge Philos. Soc. 46, 182 (1950).

${ }^{7}$ R. Kikuchi, J. Chem. Phys. 19, 1230 (1951).

${ }^{8}$ D. W. Jepsen, J. Math. Phys. 6, 405 (1965).

${ }^{9}$ J. L. Lebowitz and J. K. Percus, Phys. Rev. 155, 122 (1967).

${ }^{10}$ J. L. Lebowitz, J. K. Percus, and J. Sykes, Phys. Rev. 171, 224 (1968).

${ }^{11}$ D. G. Levitt, J. Stat. Phys. 7, 329 (1973).

${ }^{12}$ P. Kasperkovitz and J. Reisenberger, Phys. Rev. A 31, 2639 (1985).

${ }^{13}$ P. Kasperkovitz and J. Reisenberger, J. Math. Phys. 26, 2601 (1985).
${ }^{14}$ O. J. Eder and T. Lackner, Phys. Rev. A 28, 952 (1983).

${ }^{15}$ O. J. Eder and T. Lackner, Phys. Rev. A 29, 799 (1984).

${ }^{16}$ M. Robert and R. Viswanathan, J. Chem. Phys. 86, 4657 (1987); M. Robert, J. F. Jeng, and R. Viswanathan, ibid. 88, 1983 (1988).

${ }^{17}$ C. Foidi, J. Chem. Phys. 85, 410 (1986).

${ }^{18}$ T. K. Vanderlick, H. T. Davis, and J. K. Percus, J. Chem. Phys. 91, 7136 (1989).

${ }^{19}$ See, for example, J. K. Percus, J. Stat. Phys. 15, 505 (1976).

${ }^{20}$ See, for example, J. K. Percus, J. Chem. Phys. 75, 1316 (1981).

${ }^{21}$ M. E. Fisher and B. Widom, J. Chem. Phys. 50, 3756 (1969).

${ }^{22}$ H. N. W. Lekkerkerker and B. Widom, Physica A 285, 483 (2000).

${ }^{23}$ B. Widom, Science 157, 375 (1965).

${ }^{24}$ Z. W. Salsburg, R. W. Zwanzig, and J. G. Kirkwood, J. Chem. Phys. 21, 1098 (1953).

${ }^{25}$ S. Asakura and F. Oosawa, J. Chem. Phys. 22, 1255 (1954).

${ }^{26}$ J. M. Brader and R. Evans, Physica A 306, 287 (2002). 